\title{
Quality of life of breast cancer patients medicated with anti-estrogens, 2 years after acupuncture treatment: a qualitative study
}

This article was published in the following Dove Press journal:

International Journal of Women's Health

24 September 2010

Number of times this article has been viewed

Jill Hervik'

Odd Mjåland ${ }^{2}$

'Pain Clinic, Vestfold Hospital, Tønsberg, Norway; ${ }^{2}$ Department of Abdominal Surgery, Sørlandet Sykehus, Kristiansand, Norway
Correspondence: jill Hervik

Pain Clinic, Vestfold Hospital,

Tønsberg, Norway

Email jill.hervik@siv.no
Objective: The aim of this study was to examine the quality of life of breast cancer patients medicated with estrogen antagonists, 2 years after having acupuncture treatment for hot flashes.

Methods and materials: Our sample was taken from women who had recently participated in a randomized controlled trial investigating the effects of acupuncture on hot flashes, a side effect of estrogen-antagonist treatment. Forty-one women from the true acupuncture treatment group and 41 women from the control group (sham acupuncture), who had 2 years previously received a course of 15 acupuncture treatments over a period of 10 weeks, were asked to answer an open question. The question, "Would you like to share your thoughts and experiences related to your breast cancer diagnosis, treatments or anything else?" was by being open, broad, and nonspecific, intended to stimulate subjective information, which was not included in the original, or future quantitative studies. Qualitative data were analyzed using systematic text condensation.

Results: Most women were troubled by two or more side effects due to anti-estrogen medication, negatively affecting their life quality. Symptoms included hot flashes, sleep problems, muscle and joint pain, arm edema, fatigue, weight gain, depression, and lack of sexual desire. Women previously treated with sham acupuncture complained that hot flashes were still problematic, whilst those previously treated with traditional Chinese acupuncture found them less of a problem and generally had a more positive outlook on life. These results compare favorably with the findings from our original study that measured quantitatively health related quality of life.

Conclusion: Side effects due to anti-estrogen treatment seriously affect the quality of life of breast cancer operated patients. Patients who had previously been treated with traditional Chinese acupuncture complained less of hot flashes, and had a more positive outlook on life, than women who had previously been treated with sham acupuncture.

Keywords: breast cancer, anti-estrogen medication, quality of life

\section{Introduction}

The aim of our study was to examine the quality of life of breast cancer operated patients, treated with estrogen antagonists, 2 years after acupuncture treatment. By posing an open, broad, and nonspecific question about experiences related to breast cancer diagnosis and treatment, we hoped to gather subjective information from this patient group, not normally included in quantitative outcome measures.

There were around 430,000 new cases of breast cancer in Europe in 2008; 55\% of these were estrogen-receptor positive. ${ }^{1}$ Conventional medical treatment involves the use of hormone therapy for those diagnosed with estrogen-sensitive tumors, for a minimum of 5 years. Drugs include the estrogen antagonist tamoxifen and aromatase inhibitors 
such as anastrozole, exemestane, and letrozole. Many women suffer from side effects, the most common being hot flashes, affecting around $80 \%$ of women taking tamoxifen. ${ }^{2}$ The severity of hot flashes has been shown to be more severe and intense than those experienced by healthy menopausal women. ${ }^{3,4}$ The use of hormone replacement therapy, an option for healthy women, is contraindicated in estrogen-sensitive breast cancer patients. Pharmacological agents used to treat hot flashes, eg, antihypertensive clonidin, steroids, and antidepressants, often have adverse effects, such as hypotension, weight gain, and nausea respectively. ${ }^{5}$ A study from Henry Ford Hospital, Detroit randomized 50 women with breast cancer to either acupuncture or venlafaxine treatment over a period of 12 weeks; patients were followed up for 1 year. Results showed that acupuncture appeared to be as effective in reducing hot flashes as venlafaxine, an antidepressive medication. ${ }^{6}$

The authors of this study have previously carried out a randomized, controlled trial, investigating the effects of acupuncture treatment in breast cancer operated patients complaining of hot flashes. ${ }^{7}$ Results from the trial showed a significant reduction in number of hot flashes both day and night, coupled with a significant improvement in healthrelated life quality that lasted for a further 3 months post treatment in patients receiving traditional Chinese medicine (TCM) acupuncture. The only significant effect, experienced by patients in the sham acupuncture group, was a reduction in hot flashes at night during treatment; however, the effect did not last throughout the following 3 months. We concluded that acupuncture was a valuable option for this patient group suffering from side effects due to long-term anti-estrogen medication. However, the effect of acupuncture treatment beyond 3 months was not investigated.

In an attempt to accumulate qualitative information related to health and life quality from women who had been diagnosed and treated for breast cancer, the authors devised an open question: "Would you like to share your thoughts and experiences related to your breast cancer diagnosis, treatments, or anything else?" Although all patients had received acupuncture 2 years previously, the question put to them did not specifically mention acupuncture treatment. This deliberate emission was an attempt to avoid leading them in a specific direction regarding their statements, allowing them to give information important to them, whether it was centered around their breast cancer diagnosis, various treatments, social issues, or indeed other aspects of life. Acupuncture studies investigating hot flashes, in both women with breast cancer, and healthy women, have recorded changes in health outside expected outcome measures. ${ }^{8}$ We hoped that accumulation of subjective information would provide us with a broader understanding of the quality of life, including physiological, psychological, emotional, and social elements for these women. An Iranian literature review from 1982 to 2008 , examined the relationship between quality of life and survival time in cancer patients. Quality of life data were shown to be significant predictors of survival duration. ${ }^{9}$ Quality of life is a term which only became a medical issue in the 1960s, and is defined by WHO as "how an individual perceives his own life, in light of the cultural context and values of his environment, aims, expectations and worries". ${ }^{10}$ Indeed, TCM embraces these interactions. All the women included in our study had been primarily treated with traditional Western medicine; Chinese medicine was used to reduce side effects of western medication.

\section{Design, materials and methods Patients}

To gain subjective information from this patient group, a qualitative design was considered applicable. Data were gathered from participants' written answers to an open question, sent in the post, accompanied by an explanatory letter. Our sample was taken from a previous randomized controlled trial which included 59 Norwegian female patients. All patients had been recruited from The Breast Centre at Vestfold Central Hospital. Because a third of these were not reachable, a further 31 women were included; these women were comparable to the women originally included in the study, in age and in social and drinking habits. Baseline values of mean numbers of hot flashes at day and night, and total Kupperman index score, measuring healthrelated quality of life, were not significantly different, and they were treated and followed up in an identical manner to those included originally. All patients were randomized by a closed envelope technique to 15 standard treatments with TCM acupuncture or sham (minimal) acupuncture, and monitored for a further 12 weeks after completing treatment. All participants had been medicated with tamoxifen for at least 3 months, and were postmenopausal, defined as no menstruation for at least 3 months before treatment start. Patients taking medication for hot flashes either prior to or during the study were excluded. Further exclusion criteria included: previous acupuncture, simultaneous treatment with other complementary or alternative therapies, and serious systemic or psychological disorders. No hot flash severity limits were implemented. 


\section{Methods}

A total of 82 patients received an invitation to take part, and a questionnaire in the post, 2 years ( \pm 2 months) after finishing the acupuncture course of treatments. Forty-one patients had previously received TCM acupuncture, and 41 patients had received sham acupuncture (control group). Patients had been blinded to the type of treatment they had received in the original study, and had not at any point in time received any information indicating what type of treatment they had received prior to writing their statements. The study was approved by the regional committee for medical research ethics.

\section{Analysis of data}

Content analysis was done by reading and rereading the material to gain an overall impression. Simple counting of the number of women who referred to different aspects of their diagnosis, treatment, and daily life was undertaken. Groups making similar statements were indexed to develop analytical categories and were derived inductively to produce a grounded theory, developing a hypothesis from the collected data rather than defining it beforehand. To develop categories, interesting or unfamiliar terms were noted. All relevant data from each category were identified and examined using constant comparison; categories were added to reflect as many nuances in the data as possible. Categories were further refined and grouped together by using spreadsheets and the split-screen function of word-processing, creating key themes and categories for further investigation. Although researcher bias was not considered a problem, two analysts were used; neither patient identity nor previous type of treatment was revealed to the analysts. Categories were charted with entries from several respondents, and charts were mapped to define concepts and find associations between themes, with a view to providing explanations for the findings.

\section{Results}

Eight women from the original study had died during the 2 years since completing their acupuncture treatment. Twenty-one women did not return written statements. A total of 61 statements were received; 33 of these women had previously been treated with TCM acupuncture, and the other 28 had received sham acupuncture. The mean age of the participants was 51.3 (52.5 in the TCM group and 50.2 in the control group). The women provided their answers by mail. These were anonymously assessed by the first author and an oncology nurse, both experienced in research methods. The women also completed a validated Kupperman menopausal index, examining the severity of any symptoms often associated with menopause, and a questionnaire aimed at gathering information about symptom development. Patients answered five questions about: treatments in connection with their diagnosis, hot flash severity, whether they had had more acupuncture or other treatments (including medication) for their hot flash problems, and whether they still used the same estrogen-antagonist medication, had changed to another, or stopped. This data will be published at a later date in a quantitative article.

The women returned statements about their experiences relating to their breast cancer diagnosis, acupuncture treatment for hot flashes, related symptoms, and side effects of estrogen-antagonist treatment, and their daily life. There was great variation in style, and the length of their statements ranged from 0 to 364 words; the number of words in both groups was comparable. Statements were analyzed by systematic text condensation.

Although the question posed to the participants did not specifically mention hot flashes or acupuncture, 28 of the 61 participants commented on one or both. Fifteen patients, previously treated with traditional acupuncture for their hot flashes, commented on the positive effects they had experienced during and after treatment. Most went on to describe the quality and quantity of their hot flashes; 10 mentioned that they were still fewer and milder than they had been before they received treatment. Comments included: "The hot flashes have returned but only slightly at night, but they are over quickly and I have got used to them". "My family saw a change in me when the hot flashes started to lessen during acupuncture treatment, I went back to work again, my colleagues are important to me. During the last 2 years I have had some top up treatments, and will in the future if I start to get warm again". "Acupuncture helped a lot, my hot flashes were reduced to a level that I felt that I could manage, and have stayed like that". A majority of these women went on to describe how they approached, or managed their hot flashes; techniques included avoiding situations facilitating their hot flashes, such as stress, certain foods and alcohol. Five women mentioned simple relaxation techniques as a means of stopping, controlling, or shortening hot flashes. Thirteen patients from the control group commented on acupuncture and level of hot flashes, though less favorably than the TCM group. Nine women complained of severe hot flashes, only one said that acupuncture had reduced her hot flashes, four said that acupuncture had not worked, and one commented that it was painful. A typical comment was: "I have strong and frequent hot flashes, about once an hour, I wake up 4-5 
times at night, even so, the hot flashes are worse during the day". Another participant wrote: "I sweat a lot and have lots of hot flashes every day, but have learnt to tackle them, they don't bother me as much, acupuncture did not work for me". One lady related her hot flashes to stress at work, she wrote: "I work full time as a restaurant manager, I have lots of hot flashes, especially when I am stressed, I start to get warm, sweat and feel sick". Four women mentioned that food and drink affects them: "I get instant hot flashes from fatty foods, caffeine and red wine". Another woman wrote, "I found out that strong, spicy food, red wine, and chocolate provokes hot flashes". One lady commented, "If I lead a regulated life and do not eat chocolate, I feel better, and my hot flashes are not as bothersome, but it is not easy, I have not got much will power".

Only a total of five women mentioned their social and family lives, but only to demonstrate the strength of their symptoms; all but one were from the control group. One lady told how she disturbed her husband at night when she could not sleep; another described how shocked her friends were when she had a hot flash and sweat dripped down her face. One lady described how pleased she was to meet and bond with other young breast cancer patients; she felt that they understood each other's problems when she attended a rehabilitation centre.

A total of 20 women, equally divided between the two groups, described how they discovered their breast tumor and their experiences with the health service. A young woman of 30 wrote: "When I was diagnosed, I decided that I would be in control of the disease, not the other way around. I have always been mentally strong and that has not changed". Another wrote: "When I was diagnosed, I was not afraid, I felt safe at the hospital. The whole process has enriched my life. Although tamoxifen took all my strength and gave me enormous aches and pains, these disappeared when I changed to Arimidex [letrozole]. I can't complain, I have a few aches here and there, just like other women of my age". Another said: "I scratched my breast one day and felt a lump. Four weeks later I was operated, I tolerated the post-operative treatment well. I tell my friends that I am not sick, I just have a few problems". Most women who mentioned the health service seemed pleased with the service and personnel. Only two complained, both about their general practitioners; they both said that their doctors did not understand their complaints of side effects due to anti-estrogen medication and did not have time to listen to them. Another wrote that she was comforted when the surgeon said pre-operatively that she would be totally healthy again, she also said that she had confidence in all the hospital personnel. One participant wrote: "Being diagnosed with breast cancer was a shock, but much worse were the side effects of tamoxifen, hot flashes 3-4 times an hour, day and night, I was tired out, my quality of life was zero. Thanks to my doctor at the breast centre I was referred to acupuncture treatment, my hot flashes gradually reduced and I got some sleep".

A lot of women, more than $50 \%$ from both groups, wrote about side effects of their estrogen-antagonist medication. Apart from hot flashes and sweating, sleep problems and tiredness were a big problem, followed by arm edema, muscle and joint pain, body weight increase, headaches, and dry vaginal mucus membranes. One young lady wrote: "I have always been strong and in good shape, but not anymore. My body aches, in a way I cannot describe, my joints ache. My fingers are stiff, it takes time for them to warm up and they stiffen quickly when I use them". Many women used the word exhausted, a total of 16 patients either complained of sleep problems or of being tired. "I am weary and tired all the time, even though I sleep for a few hours after work". Another stated: "Hot flashes, edema in my arm, sleep problems and tiredness has reduced my quality of life and ability to work". One tired lady wrote: "I am exhausted, I did not receive any information on how the side-effects of the chemotherapy and medicines would affect me, I have had a hard time explaining these problems to the social services and my work place. As far as I know, these side-effects have not been documented, they need to be. I am a single mother and have had to tackle periods of depression, lack of sleep, hot flashes and fatigue alone, it has been really hard". Vaginal dryness, a side effect of estrogen reduction was mentioned by several women. One wrote: "It is very uncomfortable, my vagina is dry, I have had numerous urine infections, and I bleed when I have sex". Another lady wrote, "my sex life is over!" A third lady commented: "I have lost my sex drive, my husband and I are not as close now as we used to be, I put this down to lack of intimacy". A total of 20 women mentioned more than two side effects in their statements, indicating that these problems are of great importance to them.

On a positive note, many women had come to terms with their situation; many commented that despite their health problems, they were happy and content. A total of 18 patients (13 from the traditional acupuncture group and five from the control group) ended their statements on a happy note. Comments included: "I am OK. I am certain that I have received the best treatment in the world", "Despite my breast cancer diagnosis, my life is positive, I am fine", "I am healthy, the only thing I cannot do is go topless on the beach". "I have 
learnt to live with my problems. I cannot complain, it could be much worse. I am good". "Ok, so I have lost my sex drive, got gum disease and tendinitis, but I am alive and happy".

\section{Discussion}

Our results present information describing quality of life, coping mechanisms, and experiences in women operated for breast cancer. Women made statements about their situation in response to an open question 2 years after finishing acupuncture treatment (TCM or sham) for hot flashes, a side effect of anti-estrogen medicines. The variety of the written statements made by the women, although unstructured, included similar consistent themes, indicating that the question had been understood adequately. By posing such an open question, and consequently receiving unstructured statements, categorizing proved problematic. However, themes were established by repeated statements about the same subjects, but with some variation. In the original acupuncture trial 2 years previously, participants were randomized to treatment with TCM acupuncture and sham acupuncture (control). Those originally included in the TCM group who mentioned hot flashes and/or acupuncture in their statements, did so favorably, reporting fewer hot flashes, better hot flash management, and a positive attitude to acupuncture. Those who had originally been included in the control group complained more of the severity and frequency of their hot flashes; their attitude to acupuncture was generally less positive than the other group. Since acupuncture intervention reduced the amount of hot flashes significantly in the TCM group, and showed only minimal effect in the control group 2 years earlier, it is not surprising that participants from the TCM group view acupuncture more favorably than those from the control group. But what of their hot flash complaints and problems? The women from the control group reported either more frequent and/or more intense hot flashes than the other group, or at least they experienced and understood their symptoms as severe. Could it be possible that attitude to a treatment and previous experience can affect symptoms and how we tackle them at a later date? Is it feasible to expect that a series of acupuncture treatments still has effect 2 years later? Long-term follow up studies are few and inconclusive. A study from Tromsø University examining the effect of acupuncture 6 and 12 months after treatment in healthy menopausal women could not demonstrate any long-term effect. ${ }^{11}$ Frisk et al, however, could demonstrate a reduction in number of hot flashes up to 24 months after electro-acupuncture treatment in breast cancer operated women. ${ }^{12}$ An explanation for the difference between the two groups in our study could be that the significant effects of acupuncture in the TCM group gave the women a better start, by reducing hot flashes and bettering sleep quality during the first few months of antiestrogen treatment.

Despite access to various cancer groups and societies, some women felt the need to talk about their diagnosis and time in hospital. We would not immediately have expected so many women to describe these experiences. Could there be a need for these women to get it off their chests? Might they have considered the questionnaire as someone to talk to or a good listener? Is it possible that some of these women have not talked to anyone about their experience in hospital before? Or do these women have an insatiable need to talk about their situation? Busy oncology departments in Norway do not have the luxury of a psychologist or even appointments meant solely for talking or discussion, general practitioners offer 15-minute appointments, an inadequate amount of time for an in-depth conversation. However, nearly all were positive to the health service; they communicated that they had confidence in and trusted the healthcare professionals that they came into contact with. General emotional and informational support by health professionals was shown to have a direct effect on quality of life in a study of 250 Chinese breast cancer patients. ${ }^{13}$

Nearly half of the women in our study commented on symptoms they consider to be side effects of anti-estrogen medication. The recommended duration of hormonal therapy is 5 years. With such a long course of therapy, adverse effects can cause a major decrease in the quality of life. In a bibliographic review of literature covering all publications that appeared in English language in biomedical journals between 1974 and 2007, Montazeri examined the quality of life in breast cancer patients. The literature indicated that adjuvant hormonal therapies negatively affected quality of life; symptoms included arm pain, fatigue, and postmenopausal problems. Psychological anxiety and depression were common among breast cancer patients, even years after the disease diagnosis and treatment. ${ }^{14}$ The statements written by the women in our study confirm these findings and range of symptoms. Our original study showed a clear relationship between hot flashes, sleep problems, and depression. Acupuncture had a significant effect on all these symptoms. Only patients medicated with tamoxifen were included in the original study. In line with international guidelines, postmenopausal women switch to an aromatase antagonist after 2 years. Forty-two percent of the women (19\% in the TCM group and $23 \%$ in the control group) had, during the 2-year gap between the time they finished treatment and the time they made their statements, switched to an aromatase 
antagonist. A study from the University of Sussex examined the quality of life of women with breast cancer, medicated with either tamoxifen, anastrozole (aromatase antagonist), or a combination of the two. A gradual improvement in quality of life during a 2-year period was reported by over 800 women. Endocrine symptoms increased between baseline and 3 months for all groups, and stabilized thereafter. Patients taking anastrozole only, reported significantly fewer cold sweats and vaginal discharge, but more vaginal dryness, painful intercourse, and loss of sexual interest, than those taking only tamoxifen. ${ }^{15}$ Garreau et al found, by questioning over 300 women, that those taking an aromatase inhibitor suffered more from musculoskeletal problems and were more likely to switch therapy than those taking tamoxifen. ${ }^{16}$ Side effects of estrogen-antagonist treatment affect many aspects of these women's lives. These problems need to be addressed. Many women complain of not being able to move forward in their lives, being constantly reminded of their cancer diagnosis by irritating symptoms. A comfort to some will be evidence from the University Hospital, Groningen, The Netherlands, indicating that symptoms decreased significantly after discontinuation of tamoxifen, although hot flashes, sleep disturbances, and vaginal dryness persisted in those treated with high-dose chemotherapy. ${ }^{17}$

The findings in this study represent life experiences associated with breast cancer diagnosis and treatment, in a selected group of Norwegian women. Norway is one of the world's richest countries; it provides heavily subsidized healthcare, and women's rights are strong. These findings are therefore probably not transferable to other cultures and parts of the world. In hindsight we regret not obtaining data about psychological variables, since personality traits may affect approach, expectations, and reactions to diagnosis, treatments, and coping mechanisms. Interviews, discussions, and focus group data may have provided more depth; however, this study provided us with subjective information about quality of life, as experienced by women who have undergone treatment for breast cancer. These findings reinforce our belief that the side effects of anti-estrogen therapy should receive more attention in medical worlds of both East and West. For many years, medicine in the West has focused largely on prevention, operative, and conventional postoperative treatment of breast cancer, and women have put up with adverse effects of long-term anti-estrogen medication. This study highlights the need for more quantitative and qualitative studies examining problems facing women diagnosed with breast cancer, providing solutions for evidence-based treatments, psychosocial interventions, and allocation of resources.

\section{Conclusion}

This study suggests that side effects of estrogen-antagonist treatment are a significant problem for women diagnosed with breast cancer, affecting their quality of life. Although patients who had 2 years earlier been treated with TCM acupuncture complained less of hot flashes, and generally seemed to have a more positive outlook on life, they were as troubled by other side effects as the women in the control group.

\section{Acknowledgments}

This work was supported by Vestfold Hospital (SIV), Norway, and The Norwegian Acupuncture Association (NAFO). Many thanks to Kari Herland (oncology nurse, $\mathrm{SiV}$ ) for help with analysis of results.

\section{Disclosure}

The authors report no conflict of interest in this work.

\section{References}

1. Langseth H, Gislefoss RE, Martinsen JI, et al; Cancer Registry of Norway. Cancer in Norway 2008 - Cancer incidence, mortality, survival and prevalence in Norway. Oslo: Cancer Registry of Norway 2009. Available from: http://www.kreftregisteret.no/en/General/Publications/ Cancer-in-Norway/Cancer-in-Norway-2008. Accessed 2010 Aug 21.

2. Loprinzi CL, Zahasky KM, Sloan JA, Novotny PJ, Quella SK. Tamoxifen induced hot flushes. Clin Breast Cancer. 2000;1(1):52-56.

3. Love RR, Cameron L, Connell BL, Levanthal H. Systems associated with Tamoxifen treatment in postmenopausal women. Arch Intern Med. 1991;151:1842-1847.

4. Ilnyckyj A, Shanahan F, Anton PA, Cheang M, Bernstein CN. Quantification of the placebo response in ulcerative colitis. Gastroenterology. 1997;112:1854-1858

5. Hervik J, Mjaland O. Acupuncture for the treatment of hot flashes in breast cancer patients, a randomized, controlled trial. Breast Cancer Res Treat. 2009;116:311-316.

6. Hickey M, Saunders C, Partridge A, et al. Practical clinical guidelines for assessing and managing menopausal symptoms after breast cancer. Ann Oncol. 2008;19:1669-1680.

7. Walker EM, Rodriguez AI, Kohn B, et al. Acupuncture versus Venlafaxine for the management of vasomotor symptoms in patients with hormone receptor-positive breast cancer: a randomized controlled trial. J Clin Oncol. 2010;1;28(4):634-640.

8. Alraek T, Malterud K. Acupuncture for menopausal hot flashes: a qualitative study about patient experiences. J Alt Comp Med. 2009;15(2):153-158.

9. Montazeri A. Quality of life as prognostic indicators of survival in cancer patients: an overview of the literature from 1982 to 2008. Health Qual Life Outcomes. 2009;23(7):102.

10. Bonomi AE, Patrick DL, Bushnell DM, Martin M. Validation of the United States' version of the World Health Organization quality of life (WHOQOL) instrument. J Clin Epidemiology. 2000;53:1-12.

11. Borud EK, Alraek T, White A, Grimstad S. The acupuncture on hot flushes among menopausal women (ACUFLASH) study: observational follow up results at six months. Menopause. 2010;17(2): 262-268.

12. Frisk J, Carlhåll S, Kålstrøm AC, Lindh-Astrand L, Malmstrøm A, Hammer M. A long term follow-up of acupuncture and hormone therapy on hot flushes in women with breast cancer: a prospective, randomized, controlled, multicentre trial. Climacteric. 2008;11(2):166-174. 
13. Wong WS, Fielding A. Change in quality of life in Chinese women with breast cancer: changes in psychological distress as a predictor. Support care cancer. 2007;15(11):1223-1230.

14. Montazeri A. Health-related quality of life in breast cancer patients: a bibliographic review of the literature from 1972 to 2007. J Exp Clin Cancer Res. 2008 Aug 29;27:32.

15. Cella D, Fallowfield L, Barker P, Cuzick J, Locker G, Howell A; for the ATAC Trialistsa9 Group. Quality of life in the ATAC ("Arimidex", tamoxifen alone or in combination) trial after completion of five years" adjuvant treatment for early breast cancer. Breast Cancer Res Treat. 2006;100(3):273-284.
16. Garreau JR, Delamelena T, Walts D, Karamlou K, Johnson N. Side effects of Aromatase inhibitors versus Tamoxifen: the patients' perspective. Am J Surg. 2006;192(4):496-498.

17. Mourits MJ, Böckermann I, de Vries EG, et al. Tamoxifen effects on subjective and psychosexual well-being, in a randomised breast cancer study comparing high-dose and standard-dose chemotherapy. Br J Cancer. 2002;86(10):1546-1550.

International Journal of Women's Health

\section{Publish your work in this journal}

The International Journal of Women's Health is an international, peerreviewed open-access journal publishing original research, reports, reviews and commentaries on all aspects of women's healthcare including gynecology, obstetrics, and breast cancer. Subject areas include: Chronic conditions (migraine headaches, arthritis, osteoporosis);

\section{Dovepress}

Endocrine and autoimmune syndromes; Sexual and reproductive health; Psychological and psychosocial conditions. The manuscript management system is completely online and includes a very quick and fair peer-review system. Visit http://www.dovepress.com/ testimonials.php to read real quotes from published authors.

Submit your manuscript here: http://www.dovepress.com/international-journal-of-womens-health-journal 